Pure and Applied Mathematics Quarterly

Volume 6, Number 1

(Special Issue: In honor of

John Tate, Part 2 of 2$)$

$1-20,2010$

\title{
Recent Progress and Open Problems in Function Field Arithmetic — The Influence of John Tate's Work
}

\author{
Dinesh S. Thakur*
}

\begin{abstract}
The goal of this paper is to give a quick survey of some important recent results and open problems in the area of function field arithmetic, which studies geometric analogs of arithmetic questions. We will sketch related developments and try to trace the multiple influences of works of John Tate in this context.
\end{abstract}

keywords: Tate, function field, Drinfeld modules, elliptic curves, zeta values.

This paper is dedicated to my teacher John Tate. I am glad and honored to be invited to contribute to this special volume celebrating his 80th birthday. The goal of this paper is to give a quick survey of some important recent results and open problems in the area of function field arithmetic, which studies geometric analogs of arithmetic questions. We will sketch related developments and try to trace the multiple influences of works of John Tate in this context. We mainly, but not fully, limit ourselves to topics where these are clearly visible. Also, we focus mainly on results simple to state, and leave variants or generalizations to the references. General references for background on recent results in function field arithmetic are [Ros02, Gos96, $\mathrm{G}^{+} 92, \mathrm{G}^{+} 97$, Tha04].

Received November 13, 2006.

*Supported in part by NSF grant. 
Tate's fundamental contributions to the foundations of function field studies (sometimes in the context of global fields and sometimes over more general base fields) and of non-archimedean studies have been very influential in the development of number theory. Let us quickly recall some of them:

- Work with Artin on the function field basics and on the foundations of global class field theory,

- Galois cohomology of global fields,

- Study of local constants in global context,

- Study of Milnor ring in global context,

- Work on theorems of Luroth, Castelnuovo-Severi,

- Work on genus change in inseparable extensions of function fields,

- Novel treatment of residues and Riemann-Roch,

- Tate conjectures and function field analog of Birch and Swinnerton-Dyer conjectures,

- Rigid analysis in non-archimedean setting,

- Lubin-Tate's treatment of local class field theory,

- Tate elliptic curves,

- Tate-Shafarevich elliptic curves giving arbitrary large Mordell-Weil rank over global function fields,

- Tate's formulation of the Stark conjectures in general global setting and his proof of Stark-Stickelberger in the function field case,

- Conjectures of Mazur-Tate-Teitelbaum about $p$-adic Birch and SwinnertonDyer and Mazur-Tate refined conjectures in various global settings.

I have omitted specific references, as they can be easily found out from his bibliography obtainable, e.g., from MathSciNet.

It is hard to trace the influence of Tate's work on other works accurately, as the basic objects, ideas and theorems that he introduced have permeated (and been generalized by others in well-developed standard theories) throughout mathematics as the following common terms show: Tate modules, Tate elliptic curves, Tate uniformization, Tate's $q$, Lubin-Tate theory, Honda-Tate theory, Sato-Tate conjecture, $p$-divisible or Barsotti-Tate groups, Hodge-Tate decomposition, Tate cohomology, Poitou-Tate duality, Serre-Tate parameters, Tate cycles, Tate conjectures, Mumford-Tate group, Mumford-Tate conjectures, Tate twists, Tate motives, Tate spaces, Tate ring, Tate algebra, Tate residue, Cassels-Tate pairing, 
Neron-Tate heights, Shafarevich-Tate groups, Koszul-Tate resolution (complex, derivation) etc.

\section{Class field theory and Langlands Conjectures}

We have already mentioned important contributions of Tate in the 1950's to global class field theory and its cohomological aspects.

In the 1930's, Carlitz developed what is now called a Carlitz module and related analogs for $\mathbb{F}_{q}[t]$ of the exponential, $2 \pi i$, roots of unity and cyclotomic theory. His contributions were forgotten to a large extent, probably because he wrote a huge number of papers and because this theory was developed in a series of papers with uninformative titles such as 'A class of polynomials', 'A set of polynomials', 'Some properties of polynomials' etc. Carlitz's student David Hayes attended Tate's lectures at Harvard giving an exposition of explicit local class field theory by the Lubin-Tate approach of formal groups and noticed a similarity with the explicit cyclotomic approach that he learned from Carlitz. When he pointed this out, Tate encouraged Hayes to develop it further.

As Hayes writes in [Hay], while Artin, Weil and others were treating all places of function fields on equal footing, Carlitz developed his theory by singling out a distinguished place at infinity in analogy with the distinguished (archimedean this time) place at infinity for the field of rational numbers. But doing this, he missed some abelian extensions (those wildly ramified at infinity). Hayes [Hay74] then developed the full explicit class field theory for the rational function field and started considering the higher genus case.

Let $K$ be a function field of one variable over $\mathbb{F}_{q}$ of characteristic $p, \infty$ be a place of $K, A$ be the ring of elements of $K$ integral outside $\infty, K_{\infty}$ be the completion of $K$, and $C_{\infty}$ be the completion of an algebraic closure of $K_{\infty}$. We then consider $K, A, K_{\infty}, C_{\infty}$ as analogs of $\mathbb{Q}, \mathbb{Z}, \mathbb{R}, \mathbb{C}$ respectively, with $\infty$ corresponding to the archimedean place of $\mathbb{Q}$. For a finite prime $\wp$ of $A$, we can consider $A_{\wp}$ as an analog of $\mathbb{Q}_{p}$. For more on these analogies, see [Tha04, Sec. $1.1]$.

We start with the simplest case: $K=\mathbb{F}_{q}(t), A=\mathbb{F}_{q}[t]$, so that $K_{\infty}=\mathbb{F}_{q}((1 / t))$.

Let us define $C_{a}(u) \in A[u]$, for $a \in A$, by

$$
C_{1}(u)=u, C_{t}(u)=t u+u^{q}, C_{t^{n}}(u)=C_{t}\left(C_{t^{n-1}}(u)\right),
$$


and asking $C_{a}(u)$ to be $\mathbb{F}_{q^{-}}$-linear as a function of $a$. Then the $C_{a}(u)$ are analogs of the cyclotomic polynomials $u^{|n|}-1$. Thus their roots, $\zeta_{a}$ 's say, give analogs of roots of unity $\zeta_{n}$ 's. In this case,

$$
e(z)=\sum_{n=0}^{\infty} \frac{z^{q^{n}}}{\left(t^{q^{n}}-t\right)\left(t^{q^{n}}-t^{q}\right) \cdots\left(t^{q^{n}}-t^{q^{n-1}}\right)}
$$

gives an analog of the exponential function $e^{z}$ and satisfies $e(a z)=C_{a}(e(z))$ analogous to $e^{n z}=\left(e^{z}\right)^{n}$. There is $\tilde{\pi} \in C_{\infty}$, transcendental over $K$, with $e(z)=0$ if and only if $z=\tilde{\pi} a$, with $a \in A$. This thus gives analog of $2 \pi i$, because the kernel of the usual exponential is $2 \pi i \mathbb{Z}$. (Note that $\tilde{\pi}$ is well-defined up to multiplication by an element of $A^{*}=\mathbb{F}_{q}^{*}$, just as $2 \pi i$ is well-defined up to multiplication by an element of $\left.\mathbb{Z}^{*}=\{ \pm 1\}\right)$. Further, $e\left(\tilde{\pi} a_{1} / a\right)$ represent roots of $C_{a}$, for $a_{1} \in A$, just as $e^{2 \pi i k / n}$ represent roots of the $n$-th cyclotomic polynomial.

Further, we have the following analog [Hay74, Hay] of cyclotomic theory (explicit class field theory) and Kronecker-Weber theorem over $\mathbb{Q}$ :

Theorem 1. With the notation as above, if a is a non-constant polynomial in $A$, then $K(e(\tilde{\pi} / a))$ is an abelian extension of $K$, with Galois group $(A /(a))^{*}$, unramified outside $\infty$ and the primes of $A$ dividing $a$. The Frobenius at prime $\wp$ of $A$ acts on the generator by applying $C_{\wp}$.

The maximal abelian extension of $K$ can be obtained as the compositum of all such extensions together with those obtained by doing the same procedure with $\mathbb{F}_{q}[1 / t]$ replacing $A=\mathbb{F}_{q}[t]$.

For a proof, more details and generalization to any $K$, see [Gos96, Tha04, Hay79, Hay85, Hay].

Around the same time, Drinfeld, again unaware of Carlitz work, but using ideas of Lubin-Tate and taking a clue from Deligne's work relating Galois representations to modular forms, developed [Dri74], what are now called Drinfeld modules, in the setting of any function field $K$ and any place at infinity (considered as an analog of $\mathbb{Q}$ or a totally imaginary field with unique archimedean infinity, where we have explicit class field theory classically). He developed an attack not only on class field theory (which corresponded to the case of rank one), but also on $G l_{n}$ Langlands conjecture analogs. (Deligne observed that the modularity theorem in the function field case, now connecting elliptic curves over function fields to the modular curves of Drinfeld modules, follows by Drinfeld's work combined with 
previous works. See [Tha04, Pa. 215] for a sketch and note, in particular, that one step is Zarhin's proof of Tate's isogeny conjectures for abelian varieties over function fields.) Lafforgue [Laf02] finally succeeded in proving these, building on this work of Drinfeld and of many others.

Theorem 2. Let $K$ be a function field of characteristic $p$. Fix a prime $l \neq p$ and an integer $r \geq 1$. Let $A_{K}$ denote the adele ring of $K$ and $G_{K}$ be the absolute Galois group of $K$.

Then there is an explicit bijection $\pi \rightarrow \sigma(\pi)$ between (i) the set of irreducible cuspidal automorphic representations $\pi$ of $G l_{r}\left(A_{K}\right)$ which have central character of finite order, and (ii) the set of of irreducible representations $\sigma: G_{K} \rightarrow G l_{r}\left(\overline{\mathbb{Q}_{l}}\right)$ unramified outside a finite set of primes and with determinant of finite order, with the property that Frobenius and Hecke eigenvalues (or equivalently the local $L$ factors) of $\pi$ and $\sigma(\pi)$ match.

In addition to influence of Lubin-Tate work, Drinfeld's work also used ideas of rigid analysis, Tate curves, Tate uniformization, Honda-Tate theory, and Tate modules. (Note that Tate modules of Drinfeld modules give $G l_{n}\left(A_{\wp}\right)$ representations in contrast to $G l_{2}\left(\mathbb{Q}_{l}\right)$ representations given by elliptic curves over function fields). Tate's results on local-global analysis of $L$-functions, and local constants also play an important role in the Langlands program.

Gaitsgory and de Jong, Böckle-Khare proved (see [Kha] for details and references) the function field analog of Serre's conjectures that a continuous, absolutely irreducible, $n$-dimensional representation over a finite field of characteristic $l \neq p$, of the fundamental group of a geometrically irreducible smooth curve over a finite field of characteristic $p$, is automorphic. Because of Lafforgue's theorem this reduces to appropriate lifting theorems.

On the other hand, over $\mathbb{Q}$, works of Hida, Ribet and especially of Wiles, Taylor and others settling the modularity conjecture of Shimura-Taniyama-Weil, opened up powerful methods of attack in the area of the original Langlands' and Serre's conjectures. These works together with those of Böckle, Dieulefait, Khare, Kisin, Taylor, Ramakrishna, Winterberger and many others have helped to settle most of the original Serre conjectures. The level one case due to Khare [Kha06] has just been published and the earlier manuscripts of Khare and Winterberger as well 
as those announcing the odd conductor case are available on the archives. For general account and references, we refer to Khare's excellent survey paper [Kha].

In the beautiful inductive method used for this proof, Tate's 1973 proof [Tat94b] of $p=2$ case of Serre's conjectures forms the base case. Also, $p=3$ case done by Serre by similar discriminant bound methods and other works using this method of Tate are heavily used. Other influences of Tate's foundational works are seen [Kha] in uses of Barsotti-Tate representations, Hodge-Tate theory, Poitou-Tate exact sequences. Schoof's extensions of Fontaine's famous result that there is no abelian variety over $\mathbb{Z}$ played an important role in Khare's work. See [Ogg66] for Tate's earlier observation and elementary proof of the one dimensional case of elliptic curves.

\section{ZETA FUNCTIONS: ARITHMETIC OF SPECIAL VALUES}

Consider $A=\mathbb{F}_{q}[t]$ case again. Let $A+$ and $\mathbb{Z}+$ denote the set of monic polynomials and of positive integers respectively. Consider Carlitz zeta values

$$
\zeta(s)=\sum_{a \in A+} \frac{1}{a^{s}} \in K_{\infty}, \quad s \in \mathbb{Z}+
$$

While $\zeta(1)$ makes sense, and $\zeta(s p)=\zeta(s)^{p}$, in contrast to the Riemann zeta function situation, Carlitz proved (analog of Euler's result on Riemann zeta values) that the values of $\zeta$ at 'even' positive integers $s$ are rational multiples of $\tilde{\pi}^{s}$, where 'even' now means a multiple of $q-1$, which is a cardinality of $A^{*}=\mathbb{F}_{q}^{*}$, analogous to $\mathbb{Z}^{*}=\{ \pm 1\}$. In particular, they are transcendental, as $\tilde{\pi}$ is transcendental.

The nature of Riemann zeta values at odd positive integers is still a mystery. On the other hand, we have

Theorem 3. If $s$ is a positive integer, then $\zeta(s)$ is transcendental. If, further, $s$ is 'odd', i.e., not divisible by $q-1$, then $\zeta(s) / \tilde{\pi}^{s}$ as well as $\zeta_{\wp}(s)$ are transcendental, where $\zeta_{\wp}$ is the interpolation due to Goss of $\zeta$ at prime $\wp$ of $A$.

How was this proved? Greg Anderson developed higher dimensional generalization ' $t$-motives' of Drinfeld modules, put them in a framework analogous to 
motives and constructed $C^{\otimes s}$, the $s$-th tensor power of the Carlitz motive (corresponding to the Carlitz module above). This Carlitz-Tate motive is analog of the Tate twist $\mathbb{Z}(s)=\mathbb{Z}(1)^{\otimes s}$ and has corresponding exponential and logarithm attached to it. In [AT90], we constructed an algebraic point (torsion if and only if $s$ is 'even') on $C^{\otimes s}$ and expressed $\zeta(s)$ in terms of its logarithm. An analog of the Hermite-Lindemann theorem on transcendence of logarithm values (and its $\wp$-adic incarnation) was then proved [Yu91] by Jing Yu, which implied the theorem above.

In [Tha04] multizeta values were defined by

$$
\zeta\left(s_{1}, \cdots, s_{k}\right)=\sum_{\left|n_{1}\right|>\cdots>\left|n_{k}\right|} \frac{1}{n_{1}^{s_{1}} \cdots n_{k}^{s_{k}}}
$$

(where $n_{i} \in A+$ ) and their properties, relations between them and interpolations were studied.

Theorem 4. [APT] The multizeta values defined above are periods of explicit iterated extension of $C^{\otimes s_{i}}$ 's and thus transcendental.

The relations satisfied by them are under investigation. These relations are not quite the classical sum-shuffle and integral-shuffle relations and involve subtle 'digit phenomena'.

Further in $[\mathrm{AT}]$, the analogs of Ihara power series, Deligne-Soule cocycles and higher circular units are constructed. Ihara power series occurs in GrothendieckIhara program of the study of absolute Galois group over $\mathbb{Q}$ by realizing it in the automorphism group of the algebraic fundamental group of the projective line minus three points. When we consider the more manageable nilpotent quotient of the fundamental group, the mixed motive structure of iterated extensions of Tate twists shows up, with multizeta values occurring in the DeRham-Betti aspects and Ihara power series and Deligne-Soule cocycles occurring in the etale aspects of the meta-abelian simplification. We have these ingredients, as we have the mixed Tate-motives theory in function fields, but we have no good understanding of the fundamental group background yet!

David Goss (see [Gos96]) interpolated the zeta values at positive integers above to a much larger continuous space containing, in particular, the negative integers. (Cohomological aspects of $L$-functions in this situation have been developed by Taguchi, Wan [TW96], Pink and Böckle [Böc02]). The special values at positive 
as well as negative integers are related in a way analogous to the Herbrand-Ribet theorem to class groups of cyclotomic extensions, with the values at positive integers linking to the class groups of integral closures of $\mathbb{F}_{q}[t]$ in the cyclotomic extensions, while the values at the negative integers linking to the full $P i c^{0}$ of the cyclotomic extensions. But still no functional equation connecting the two sets of values is understood in contrast to the Riemann zeta function functional equation whose nice analysis was done in Tate's thesis. We do have good analogs [Tha04] of Gamma functions, but do not understand them as a factor at infinity for zeta as in Tate's thesis.

Classically, a simple analysis of poles of gamma factors occurring in the functional equations of general zeta functions leads to determination of orders of vanishing of zeta functions at negative integers. In function fields, the story is not even conjecturally fully understood. There is some interesting systematic 'extra vanishing' phenomena of zeta at $-s$ depending on digit combinatorics of $s$ and Weierstrass gaps [Tha04, Sec. 5.4] and [Gos].

\section{Zeta functions: Stark Conjectures}

Tate generalized Brumer and Stark conjectures (giving ideal class annihilators and abelian extensions via analytic processes in the ground field) to global settings, considered refined conjectures and systematically worked out many basic functorialities and results on them.

For a finite abelian extension $L$ of a global field $K$ with Galois group $G$ and a non-empty set $T$ of places of $K$ containing at least all those ramified in $L$, by character/Fourier theory, there is a unique $\theta=\theta_{T, L} \in \mathbb{C}[G]$ such that

$$
\psi(\theta)=L_{T}(0, \bar{\psi})
$$

for all complex valued characters $\psi$ of $G$ (extended linearly to the group algebra), where the $L$ function

$$
L_{T}(s, \psi):=\prod_{\wp \notin T}\left(1-\psi\left(\operatorname{Frob}_{\wp}\right) \operatorname{Norm}(\wp)^{-s}\right)^{-1}, R e(s)>1
$$

is a rational function in $q^{-s}$ and is finite at 0 . Let $\mu$ be the number of roots of unity in $L$ and $\omega:=\mu \theta$.

Theorem 5. (Tate and Deligne [Tat84]) Let $K$ be a function field and $P$ any prime divisor of $L$. We have 
(1) $\omega \in \mathbb{Z}[G]$,

(2) If $|T| \geq 2$, then $P^{\omega}$ is a principal divisor $(\ell)$ of some $\ell \in L$,

(3) If $T=\{v\}$, then $P^{\omega}$ is $(\ell)+n v_{L}$, for some $\alpha \in L$ and $n \in \mathbb{Z}$ and where $v_{L}$ denotes the simple sum of places of $L$ above $v$.

(4) If $\lambda^{\mu}=\ell$, then $L(\lambda)$ is abelian over $K$.

Let us give a quick sketch of Tate's nice proof of (2) for a geometric extension of a function field (an analog of the Stickelberger theorem on ideal class annihilators): The class group of a curve is the group of $\mathbb{F}_{q}$-rational points i.e., the part of the $\overline{\mathbb{F}_{q}}$-points of its Jacobian where the Frobenius $F$ acts as the identity, whereas its $L$-function is essentially the characteristic function of the Frobenius on the corresponding component of the Jacobian or rather the Tate module. Hence it kills the component when $t=F$ by the Cayley-Hamilton theorem. Hence $\omega$ which is just a linear combination of $L$-values at $s=0$ (i.e. $t=1$ ) kills the class group. (See [Tha04] and references there for more details.) Using one-motives in place of Jacobian, Deligne generalized from a geometric extension to any extension and proved the theorem above.

These ideas inspired the proof [GS85] of the Herbrand-Ribet analog (using the zeta values at negative integers) that was mentioned in the last section

Tate then suggested to Hayes that his Drinfeld module work might imply another proof of this theorem giving $\lambda$ (as in the Theorem) explicitly (after the reduction technology developed in [Tat84] is used). This was proved in [Hay85]

In 1987, Mazur and Tate [MT87] developed a refinement of $L$-function conjectures in the case of elliptic curves. They ran a seminar at Harvard working out these ideas in various situations, where Gross and Hayes worked out (and Hayes proved [Hay88]) the refined $p$-adic abelian Stark conjectures in the number field and the function field case respectively. See works of Ki Seng Tan and Joongul Lee for some results on the refined elliptic curves case.

In a very interesting recent work, Greg Anderson has a refinement of the Stark conjectures [And06] (see also his recent preprints for proofs using Lang-Serre geometric class field theory and Drinfeld's shtukas) which involves two rather than one variable algebraic functions. This generalizes his earlier work [And] in the $\mathbb{F}_{q}[t]$ case. For a long time, examples suggested existence of a generalization, 
but a good formulation was not at all clear. Anderson generalizes to higher genus (see also [And94]) by using an adelic harmonic analysis formulation in the language and framework of Tate's thesis.

\section{Diophantine Geometry}

Tate's foundational work developing tools of group schemes, Tate modules, $p$ divisible groups, Hodge-Tate decompositions, Neron-Tate heights, $p$-adic sigma functions, Tate curves (with their place in moduli studies) etc. has played an important role in Diophantine geometry over global fields. Works of Serre, Mazur, Faltings, Fontaine and others made heavy use of these tools.

Tate's conjectures and his results on them have also been very influential. For a nice presentation of the precise statements and of the connections between Tate conjectures relating algebraic cycles on varieties over fields finitely generated over the prime fields, galois invariant cohomology classes, order of poles of their zeta functions, Tate conjectures on isogenies of abelian varieties, Tate's analogs of conjectures of Birch and Swinnerton-Dyer (BSD) in the function field case, as well as for the summary of evidence, see [Tat94a].

Tate proved the Tate conjecture for abelian varieties over finite fields basically via a Shafarevich type finiteness result (trivial over finite fields). Faltings reversed the direction to deduce the Shafarevich finiteness conjecture from his Tate's conjecture proof, which in turn was modelled on Tate's original proof together with Zarhin's ideas which proved Tate conjectures for function fields. Faltings then deduced Mordell's finiteness conjecture from the Shafarevich conjecture using ideas of Pashin in Parshin's proof of the Mordell conjecture over function fields.

In many respects, Drinfeld modules (of rank two, for best analogies) are analogs of elliptic curves. Their higher dimensional generalizations, such as $t$-motives or $A$-motives, are analogs of abelian varieties (or of motive representing their $H_{1}$ ). Drinfeld modules also have 'characteristic' which can be generic (analog of characteristic zero) or finite prime $\wp \in A$, and we can consider Drinfeld modules in finite field, local or global setting. Thus many theorems or conjectures about abelian varieties have two different analogs in function fields: in terms of abelian varieties over function fields or in terms of $t$-motives. 
In addition to proofs of Tate, Zarhin, Faltings respectively, over finite fields, function fields, number fields respectively of Tate conjectures mentioned, let us look at the function field situation in this $t$-motive realm:

For Drinfeld modules over finite fields, an analog of the Tate isogeny theorem was proved [Dri77] by Drinfeld. For Drinfeld modules of generic characteristic (in fact, in a much more general setting), the analog of the Tate conjecture/Faltings theorem was established by Tamagawa and Taguchi [Tam94, Tag95, Tag96]. This was done by a method (quite different from Faltings in the classical case) inspired by the previous important work of Anderson [And93], where he proves an analog of Tate conjectures for 'formal $t$-modules', by approximating solutions of ' $\wp$-adic linear Frobenius equations'. Taguchi [Tag93, Tag91] also proved the semisimplicity of the Galois representation on the Tate module, for both finite and generic characteristic Drinfeld modules. Taguchi [Tag99] proved that a given $L$-isogeny class of Drinfeld $A$-module contains only finitely many $L$-isomorphism classes, for $L$ a finite extension of $K$. Oliver Watson has recently proved an analog of the Tate conjecture in the equi-characteristic case, in his 2003 University of Pennsylvania thesis.

Classically, there is a well-known theorem of Serre on the image of the Galois representation (with the general conjecture for abelian varieties being that of Mumford-Tate) obtained from the torsion of elliptic curves. Pink [Pin97] showed that if $\rho$ has no more endomorphisms than $A$, then for a finite set $S$ of places $\wp \neq \infty$, the image of $\operatorname{Gal}\left(K^{s e p} / K\right)$ in $\prod_{\wp \in S} G L_{n}\left(A_{\wp}\right)$ for the corresponding representation for rank $n$ Drinfeld modules is open. (Note that this is weaker than the Serre type adelic version, but much stronger (unlike the classical case) than the case of one prime $\wp$, because we are dealing with all huge pro- $p$ groups here, even though the primes $\wp$ change. So the simple classical argument combining $p$-adic and $l$-adic information to go from the result for one place to the result for finitely many places does not work). This has been generalized and improved in recent works of Pink, Traulsen and Gardeyn.

Let us mention that while over number fields, the Tate isogeny conjecture and the Shafarevich finiteness conjecture follow from each other for abelian varieties, it is easy to exhibit [Tha04, Sec. 6.1] infinitely many non-isomorphic rank 2 Drinfeld modules, with good reduction everywhere (so not only the support of the 
discriminant is bounded, but the discriminant is one). So with many analogies, there are a few important contrasts as well.

Finally, let us mention that the analog for Drinfeld modules of the conjecture of Tate and Voloch [TV96] (predicting existence of a lower bound for the $p$-adic distance of torsion of a semi-abelian variety over $C_{p}$ from a closed subvariety not containing it) has been recently announced in a preprint by D. Ghioca.

\section{Diophantine Geometry: Elliptic CuRVes OVer Function fieldS}

Tate's Haverford lecture notes and Inventiones survey on elliptic curves beautifully and systematically laid out the subject area and became the necessary first reading for anybody interested in learning the subject.

In a very influential Bourbaki seminar [Tat95] of 1966, Tate explained (joint work with Michael Artin) the homological machinery behind the geometric case of BSD conjectures and proved

Theorem 6. For an elliptic curve $E$ over a function field $K$, (1) its analytic rank (i.e., the order of vanishing of its $L$ function at $s=1$ ) is at least its arithmetic rank (i.e. the rank of the Mordell-Weil group of its rational points); (2) These two ranks are equal if and only if the Shafarevich-Tate group $\amalg$ is finite if and only if the $\ell$-primary part of $\amalg$ is finite for one prime $\ell$. Further, if these equivalent conditions hold, the full BSD conjecture, giving the conjectural formula for the leading term of the $L$ function at $s=1$, holds.

As stated, this uses the later refinements due to Milne and others taking care of the $p$-part, allowing $\ell=p$ and $p=2$ etc. These were not handled in [Tat95], essentially because Tate used etale cohomology and crystalline cohomology machinery needed for taking care of $p$ was developed soon thereafter. For a generalization to abelian varieties, see [Sch82].

Iwasawa used analogies between function fields and number fields to develop Iwasawa theory and carried over Hasse-Weil's geometric understanding and cohomological machinery related to zeta functions to the $p$-adic case in number fields. Transferring Tate's ideas to this realm led to the development of results on the p-adic BSD analog by Perrin-Riou [PR83], using the Iwasawa theoretic machinery. More recently, Bertiloni and Darmon [BD01] developed another analog, this 
time dealing with $p$-adic $L$ function defined analytically and using rigid analysis and $p$-adic uniformization ideas, initiated by Tate!

It suffices for our purposes below to say that in the function field case, Tate proved (see [Tat94a]) the rank equality and thus the full BSD conjecture for $E$ which is iso-trivial or $E$ whose corresponding elliptic surface over $\mathbb{F}_{q}$ is dominated by a product of curves, in particular Fermat surfaces.

Now we describe 1967 work of Shafarevich-Tate and a recent nice advance by my colleague Douglas Ulmer:

It is not known whether the (arithmetic or analytic) ranks of $E(\mathbb{Q})$ are bounded as $E$ runs through elliptic curves $E$ over $\mathbb{Q}$, the usual bet seems to be that they are not. The largest rank known today is around 28 .

In a famous paper, Shafarevich and Tate [TS67] showed that over a fixed $\mathbb{F}_{p}(t)$, there do exist elliptic curves of arbitrarily large arithmetic rank: They considered certain hyperelliptic quotient $C$ over $\mathbb{F}_{p}$ of the Fermat curve of degree $p^{n}+1$. Its zeta function can be calculated in terms of Gauss sums following Weil, and the Gauss sums were made more explicit by them for this degree. This allowed them to show that the Jacobian of $C$ has a supersingular elliptic curve $E_{0}$ as an isogeny factor to a high multiplicity $m$ over $\mathbb{F}_{p}$. (Note that this multiplicity can be calculated by Honda-Tate theory). If $E$ is the constant curve over $\mathbb{F}_{p}(t)$ based on $E_{0}$, over quadratic extension $F=\mathbb{F}_{p}(C)$ it has rank $m$, as $E(F) /$ torsion $=\operatorname{Hom}_{\mathbb{F}_{p}}\left(J(C), E_{0}\right)$. Thus the quadratic twist of $E$ by $F$ has a large arithmetic rank over $\mathbb{F}_{p}(t)$.

But the curves they exhibited are isotrivial ( $j$-invariant is in $\mathbb{F}_{q}$ ). Thus the situation is not readily comparable to the number field case. Shioda exhibited non-isotrivial elliptic curves with arbitrarily large rank over $\overline{\mathbb{F}_{q}}(t)$. Ulmer [Ulm02] showed that, in fact, they have arbitrarily large arithmetic rank over $\mathbb{F}_{q}(t)$ and proved (for a variant of equations working for all $p$ ) the following

Theorem 7. For non-isotrivial $E$ over $K=\mathbb{F}_{p}(t)$ given by $y^{2}+x y=x^{3}-t^{p^{n}+1}$, $B S D$ holds and its rank is at least $\left(p^{n}-1\right) / 2 n$. Further, the degree of the conductor of $E$ is $p^{n}+2$ or $p^{n}+4$ depending on whether $p^{n}+1$ is divisible by 6 or not.

Here is a sketch showing influence of Tate's ideas and results: Following Shioda, Ulmer gets a dominant rational map from Fermat surface of degree $p^{n}+1$ to the surface over $\mathbb{F}_{p}$ corresponding to $E$, which gives the BSD conjecture for $E$ 
by results of Tate mentioned above. By detailed analysis of the Fermat quotient isomorphic to this surface, he expresses its zeta function (related to the $L$-function of $E$ ) in terms of the zeta function of the Fermat surface. Using the ShafarevichTate calculation mentioned above, he calculates the analytic rank, which is now also the arithmetic rank. Finally, the conductor calculation is done by Tate's algorithm for minimal models!

Ulmer further showed that these curves (as well as Shafarevich-Tate isotrivial curves) asymptotically attain the upper bound for the rank in terms of the size of the conductor of the elliptic curve. This gives stronger support to the corresponding number fields conjecture predicting the existence of a family of elliptic curves $E$ with conductors $N$ tending to infinity, with rank at least $c \log (N) / \log (\log (N))$ for some $c>0$, coming from the random matrix theory analogies compared to a competing conjecture coming from probabilistic models. (In the number field case, it was shown earlier [KM00] using Heegner points that the conjecture obtained from the first conjecture by replacing elliptic curves by abelian varieties is true). Darmon produced more examples of high rank curves, by an alternate method of analysis of zeta functions, but as pointed out in [Ulm04], the calculation of root numbers he needs requires knowing the local representation of decomposition groups on the Tate module at places of bad reduction and ultimately boils down to analyzing super-singular Gauss sums using Shafarevich-Tate!

Trying to answer a question of Ellenberg about behavior of ranks as you go up a certain tower of extensions, Ulmer realized that the examples above are not really special and proved

Theorem 8. Given any non-isotrivial elliptic curve $E$ over $\mathbb{F}_{q}(t)$, there exists a finite extension $\mathbb{F}_{r}(u)$ of $\mathbb{F}_{q}(t)$ such that $E$ has unbounded analytic ranks in the tower $\mathbb{F}_{r}\left(u^{1 / d}\right)$.

(See [Ulm] for this, many more related interesting results and generalizations to abelian varieties.)

As $\mathbb{F}_{r}\left(u^{1 / d}\right)$ is isomorphic to $\mathbb{F}_{r}(x), E$ thus gives a family of curves with arbitrarily large rank over this base. Note that like isotriviality, this property of a rational function field that it contains a copy of itself in several different ways, by replacing $t$ by a rational function of $t$, is again not readily comparable to the number field case! 


\section{Diophantine Geometry: Diophantine approximation}

We just mention that in the related field of Diophantine approximation of algebraic elements over function fields, the naive analog of Roth's theorem that 'diophantine approximation exponent of algebraic real numbers is two' fails. For connections with deformation theory and many interesting recent results and open questions about distribution of Diophantine approximation exponents of algebraic elements and about their continued fraction expansions, we refer to [Tha04] and [Tha].

\section{TRAnscendence of SPECIAL VALUes AND PERIOdS}

Let us begin with a transcendence result on the period of the Tate elliptic curve:

Let $p$ be a prime number, and $k$ be an algebraic closure of $\mathbb{F}_{p}$. Let $q_{0}$ be a variable and consider $a_{4}, a_{6} \in k\left[\left[q_{0}\right]\right]$ defined by

$$
a_{4}:=\sum_{n \geq 1} \frac{-5 n^{3} q_{0}^{n}}{1-q_{0}^{n}}, \quad a_{6}:=\sum_{n \geq 1} \frac{-\left(7 n^{5}+5 n^{3}\right) q_{0}^{n}}{12\left(1-q_{0}^{n}\right)} .
$$

Theorem 9. The period $q_{0}$ of the Tate elliptic curve $y^{2}+x y=x^{3}+a_{4} x+a_{6}$ over $K:=k\left(a_{4}, a_{6}\right)$ is transcendental over $K$.

This function field analog of Mahler-Manin conjecture was proved by Voloch [Vol96], by approximating $q_{0}$ by algebraic quantities and getting a contradiction by analyzing the Galois action using Igusa's theorem.

Soon afterwards, the original conjecture was proved. See [Wal97] for the history and an account of the proof. Nice application is that ' $\log _{p} q_{0}$ ' appearing in the $p$-adic Birch and Swinnerton-Dyer conjectures of Mazur, Tate and Teitelbaum (Theorem of Stevens/Greenberg) does not then vanish, so that the order of vanishing of the $L$-functions is exactly as predicted in the conjectures.

Voloch explained his theorem and proof in a seminar at the University of Arizona and I could give another proof [Tha96] (and yet another [AT99] with Allouche) using the automata criterion for algebraicity of Christol, which says that $\sum f_{n} x^{n}$ is algebraic over $\mathbb{F}_{q}(x)$, if and only if $f_{n} \in \mathbb{F}_{q}$ is produced by a $q$ automaton, if and only if there are only finitely many subsequences of the form $f_{q^{k} n+r}$ with $0 \leq r<q^{k}$. (See [Tha04, Cha. 11] for definitions and details). 
This result on the transcendence of the Tate period was the start of my work on applications of automata theory to naturally occurring quantities in the setting of function field arithmetic, by giving refined transcendence theorems for them using results in computer science, formal language theory and logic. In my thesis, special values of arithmetic gamma for $\mathbb{F}_{q}[t]$ defined by Carlitz and Goss were calculated and related to the periods of Drinfeld modules and an analog of the Chowla-Selberg formula was proved. Thus applying the results of Jing Yu on the transcendence of periods, knowledge of transcendence of gamma values at fractions was parallel in $\mathbb{Z}$ and $\mathbb{F}_{q}[t]$ case. The automata techniques (and help from automata experts Allouche, Mendes-France, Yao) allowed me to get a complete result showing ( [Tha04, Cha. 11] for details) that all the monomials in gamma values at fractions that I had not shown to be algebraic in my thesis are, in fact, transcendental!

For the $\wp$-adic interpolation due to Goss of the gamma, Yao and myself only managed partial results [Tha04, Cha. 11], and the general case is still open.

There is another gamma function (geometric case)

$$
\Gamma(x)=\frac{1}{x} \prod_{a \in A+}\left(1+\frac{x}{a}\right)^{-1}
$$

for which there is an even more satisfying result. I had proved the functional equations for this gamma function giving algebraicity of some explicit monomials, and proved transcendence of a few (all, if $q=2$ ) values at proper fractions in $K$ by connecting them to the periods of Drinfeld modules.

By $\Gamma$-monomial we will mean an element of the subgroup of $C_{\infty}^{*}$ generated by $\tilde{\pi}$ and the values of $\Gamma$ at proper fractions in $K$.

Theorem 10. [ABP04] A set of $\Gamma$-monomials is $\bar{K}$-linearly dependent exactly when some pair of $\Gamma$-monomials is. Pairwise $\bar{K}$-linear dependence is decided by an explicit combinatorial criterion and exactly those monomials mentioned above are the only algebraic ones.

In particular, the transcendence degree of the field extension of $\bar{K}$ generated by $\tilde{\pi}$ and gamma values at proper fractions with denominators dividing $a \in A$ over $\bar{K}$ is $1+(1-1 /(q-1))\left|(A / a)^{*}\right|$. 
(We remark here that there is [Tha04, Sec. 4.12] a unified Galois-theoretic description of the 'explicit algebraic monomials' mentioned above, in both classical and function field cases, and that in the classical as well as in the geometric gamma case (but not in the arithmetic case) they follow from reflection and multiplication formulas.)

This was done by expressing the gamma monomials as periods of appropriate $t$-motives (these motives are defined using the same two variable functions mentioned in the Stark conjecture section) and by the powerful transcendence criterion of [ABP04] that all period relations come from motivic relations. This was further developed by Papanikolas [Pap] who proved the analog of the Grothendieck conjecture that the transcendence degree of the field generated by the periods of a motive is the dimension of its motivic Galois group (or MumfordTate group if considering the analogy in the abelian varieties situation), i.e., the tannakian group of the tannakian category generated by its powers. Jing Yu and Chang $[\mathrm{YC}]$ have applied this to get all algebraic dependence relations between Carlitz zeta values and we should soon have a result about multizeta values. See [Tha] for a sketch.

There are still many open questions about the nature of values of $\wp$-adic interpolations, algebraic dependence relations for the Carlitz-Goss arithmetic gamma function mentioned above as well as their generalizations to arbitrary function fields, and for another gamma function developed in [Tha04, Sec.8.3, 8.7].

Acknowledgement: I am very grateful to Jean-Pierre Serre and Greg Anderson for their detailed suggestions for improvement.

\section{REFERENCES}

[ABP04] G. Anderson, W. Brownawell, and M. Papanikolas. Determination of the algebraic relations among special $\Gamma$-values in positive characteristic. Ann. of Math. (2), 160(1):237313, 2004.

[And] G. Anderson. A two-dimensional analogue of Stickelberger's theorem. In $\left[G^{+} 92\right]$, pages $51-73$.

[And93] G. Anderson. On Tate modules of formal t-modules. Internat. Math. Res. Notices, (2):41-52, 1993.

[And94] G. Anderson. Rank one elliptic A-modules and A-harmonic series. Duke Math. J., 73(3):491-542, 1994. 
[And06] G. Anderson. A two-variable refinement of the Stark conjecture in the function-field case. Compos. Math., 142(3):563-615, 2006.

[APT] G. Anderson, M. Papanikolas, and D. Thakur. Multizeta values for $F_{q}[t]$, their period interpretation and transcendence properties. Preprint.

[AT] G. Anderson and D. Thakur. Ihara power series for $F_{q}[t]$. Preprint.

[AT90] G. Anderson and D. Thakur. Tensor powers of the Carlitz module and zeta values. Ann. of Math. (2), 132(1):159-191, 1990.

[AT99] J-P. Allouche and D. Thakur. Automata and transcendence of the Tate period in finite characteristic. Proc. Amer. Math. Soc., 127(5):1309-1312, 1999.

[BD01] M. Bertolini and H. Darmon. The $p$-adic $L$-functions of modular elliptic curves. In Mathematics unlimited-2001 and beyond, pages 109-170. Springer, Berlin, 2001.

[Böc02] G. Böckle. Global L-functions over function fields. Math. Ann., 323(4):737-795, 2002.

[Dri74] V. Drinfel'd. Elliptic modules. Mat. Sb. (N.S.), 94(136):594-627, 656, 1974.

[Dri77] V. Drinfel'd. Elliptic modules. II. Mat. Sb. (N.S.), 102(144)(2):182-194, 325, 1977.

$\left[\mathrm{G}^{+} 92\right]$ D. Goss et al., editors. The arithmetic of function fields, volume 2 of Ohio State University Mathematical Research Institute Publications, Berlin, 1992. Walter de Gruyter \& Co.

$\left[\mathrm{G}^{+} 97\right]$ E.-U. Gekeler et al., editors. Drinfeld modules, modular schemes and applications, River Edge, NJ, 1997. World Scientific Publishing Co. Inc.

[Gos] D. Goss. Zeros of $L$-series in characteristic $p$. Preprint.

[Gos96] D. Goss. Basic structures of function field arithmetic. ). Springer-Verlag, Berlin, 1996.

[GS85] D. Goss and W. Sinnott. Class-groups of function fields. Duke Math. J., 52(2):507-516, 1985 .

[Hay] D. Hayes. A brief introduction to Drinfel'd modules. In [G+92], pages 1-32.

[Hay74] D. Hayes. Explicit class field theory for rational function fields. Trans. Amer. Math. Soc., 189:77-91, 1974.

[Hay79] D. Hayes. Explicit class field theory in global function fields. In Studies in algebra and number theory, pages 173-217. Academic Press, New York, 1979.

[Hay85] D. Hayes. Stickelberger elements in function fields. Compositio Math., 55(2):209-239, 1985.

[Hay88] D. Hayes. The refined $\wp$-adic abelian Stark conjecture in function fields. Invent. Math., 94(3):505-527, 1988.

[Kha] C. Khare. Serre's modularity conjecture: a survey of level one case. To appear in Proc. of LMS Durham Conference.

[Kha06] C. Khare. Serre's modularity conjecture: the level one case. Duke Math. J., 134(3):557589, 2006.

[KM00] E. Kowalski and P. Michel. A lower bound for the rank of $J_{0}(q)$. Acta Arith., 94(4):303$343,2000$.

[Laf02] L. Lafforgue. Chtoucas de Drinfeld et correspondance de Langlands. Invent. Math., 147(1):1-241, 2002.

[MT87] B. Mazur and J. Tate. Refined conjectures of the "Birch and Swinnerton-Dyer type". Duke Math. J., 54(2):711-750, 1987. 
[Ogg66] A. Ogg. Abelian curves of 2-power conductor. Proc. Cambridge Philos. Soc., 62:143148, 1966.

[Pap] M. Papanikolas. Tannakian duality for anderson-drinfeld motives and algebraic independence of carlitz logarithms. Preprint.

[Pin97] R. Pink. The Mumford-Tate conjecture for Drinfeld-modules. Publ. Res. Inst. Math. Sci., 33(3):393-425, 1997.

[PR83] B. Perrin-Riou. Descente infinie et hauteur p-adique sur les courbes elliptiques à multiplication complexe. Invent. Math., 70(3):369-398, 1982/83.

[Ros02] M. Rosen. Number theory in function fields. Springer-Verlag, New York, 2002.

[Sch82] P. Schneider. Zur Vermutung von Birch und Swinnerton-Dyer über globalen Funktionenkörpern. Math. Ann., 260(4):495-510, 1982.

[Tag91] Y. Taguchi. Semisimplicity of the Galois representations attached to Drinfel'd modules over fields of "finite characteristics". Duke Math. J., 62(3):593-599, 1991.

[Tag93] Y. Taguchi. Semi-simplicity of the Galois representations attached to Drinfel'd modules over fields of "infinite characteristics". J. Number Theory, 44(3):292-314, 1993.

[Tag95] Y. Taguchi. The Tate conjecture for t-motives. Proc. Amer. Math. Soc., 123(11):32853287, 1995.

[Tag96] Y. Taguchi. On $\phi$-modules. J. Number Theory, 60(1):124-141, 1996

[Tag99] Y. Taguchi. Finiteness of an isogeny class of Drinfeld modules. Correction to a previous paper: "Ramifications arising from Drinfel'd modules" [in the arithmetic of function fields (columbus, oh, 1991), 171-187, de Gruyter, Berlin, 1992; MR 94b:11049]. J. Number Theory, 74(2):337-348, 1999.

[Tam94] A. Tamagawa. Generalization of Anderson's $t$-motives and Tate conjecture. Sūrikaisekikenkyūsho Kōkyūroku, (884):154-159, 1994.

[Tat84] J. Tate. Les conjectures de Stark sur les fonctions $L$ d'Artin en $s=0$, volume 47 of Progress in Mathematics. Birkhäuser Boston Inc., Boston, MA, 1984. Lecture notes edited by Dominique Bernardi and Norbert Schappacher.

[Tat94a] J. Tate. Conjectures on algebraic cycles in l-adic cohomology. In Motives (Seattle, WA, 1991), volume 55 of Proc. Sympos. Pure Math., pages 71-83. Amer. Math. Soc., Providence, RI, 1994.

[Tat94b] J. Tate. The non-existence of certain Galois extensions of $\mathbf{Q}$ unramified outside 2. In Arithmetic geometry (Tempe, AZ, 1993), volume 174 of Contemp. Math., pages 153-156. Amer. Math. Soc., Providence, RI, 1994.

[Tat95] J. Tate. On the conjectures of Birch and Swinnerton-Dyer and a geometric analog. In Séminaire Bourbaki, Vol. 9, Exp. 306 (1966), pages 415-440. Soc. Math. France, Paris, 1995.

[Tha] D. Thakur. Diophantine approximation and transcendence in finite characteristic. To appear in proceedings of International conference in Diophantine equations, DION 2005, TIFR, to be published by Springer.

[Tha96] D. Thakur. Automata-style proof of Voloch's result on transcendence. J. Number Theory, 58(1):60-63, 1996. 
[Tha04] D. Thakur. Function field arithmetic. World Scientific Publishing Co. Inc., River Edge, NJ, 2004.

[TS67] J. Tate and I. Shafarevich. The rank of elliptic curves. Dokl. Akad. Nauk SSSR, 175:770-773, 1967.

[TV96] J. Tate and J-F. Voloch. Linear forms in p-adic roots of unity. Internat. Math. Res. Notices, (12):589-601, 1996.

[TW96] Y. Taguchi and D. Wan. $L$-functions of $\phi$-sheaves and Drinfeld modules. J. Amer. Math. Soc., 9(3):755-781, 1996.

[Ulm] D. Ulmer. $L$-functions with large analytic rank and abelian varieties with large algebraic rank over function fields. To appear in Inven. Math.

[Ulm02] D. Ulmer. Elliptic curves with large rank over function fields. Ann. of Math. (2), 155(1):295-315, 2002.

[Ulm04] D. Ulmer. Elliptic curves and analogies between number fields and function fields. In Heegner points and Rankin L-series, volume 49 of Math. Sci. Res. Inst. Publ., pages 285-315. Cambridge Univ. Press, Cambridge, 2004.

[Vol96] J-F. Voloch. Transcendence of elliptic modular functions in characteristic $p$. J. Number Theory, 58(1):55-59, 1996.

[Wal97] M. Waldschmidt. Sur la nature arithmétique des valeurs de fonctions modulaires. Astérisque, (245):Exp. No. 824, 3, 105-140, 1997. Séminaire Bourbaki, Vol. 1996/97.

[YC] J. Yu and C. Chang. Determination of algebraic relations among special zeta values in positive characteristic. Preprint.

[Yu91] J. Yu. Transcendence and special zeta values in characteristic p. Ann. of Math. (2), 134(1):1-23, 1991.

Dinesh S. Thakur

University of Arizona, Tucson

E-mail: thakur@math.arizona.edu 